\title{
Forces between a single atom and its distant mirror image
}

\author{
Pavel Bushev, Alex Wilson, Jürgen Eschner*, Christoph Raab, \\ Ferdinand Schmidt-Kaler, Christoph Becher, and Rainer Blatt \\ Institut für Experimentalphysik, Technikerstr. 25, A-6020 Innsbruck, Austria
}

(Dated: October 29, 2018)

\begin{abstract}
An excited-state atom whose emitted light is back-reflected by a distant mirror can experience trapping forces, because the presence of the mirror modifies both the electromagnetic vacuum field and the atom's own radiation reaction field. We demonstrate this mechanical action using a single trapped barium ion. We observe the trapping conditions to be notably altered when the distant mirror is shifted by an optical wavelength. The well-localised barium ion enables the spatial dependence of the forces to be measured explicitly. The experiment has implications for quantum information processing and may be regarded as the most elementary optical tweezers.

PACS numbers: $42.50 . \mathrm{Pq}, 42.50 . \mathrm{Vk}, 32.80 . \mathrm{Pj}, 42.50 . \mathrm{Ct}$
\end{abstract}

An atom which sits in the vicinity of mirrors or reflectors experiences energy shifts of its electronic states. These level shifts are known as the van der Waals, Casimir-Polder [1] and resonant radiative shifts [2, 3], the latter of which is caused by a retarded interaction of the atom with its own radiation field. For an atom in its excited state and at distances from a mirror much less than the transition wavelength, the level shift will be dominated by the van der Waals interaction, whilst in the far field the level shift is attributed to the resonant interaction with its own reflected field 3, 4, 5, 6]. Such far-field shifts have been observed with an atomic beam traversing an optical resonator [7] and with atoms in a microwave cavity [8]. The same effect has been predicted for a single trapped ion whose emitted radiation field is reflected back by a single, distant mirror [9], and recently this level shift has been observed with an indirect spectroscopic method [10].

The far-field mirror-induced shift of an excited atomic level oscillates on the wavelength scale when the atommirror distance is varied. Therefore, when the position of the atom is controlled to the extent that it becomes sensitive to this spatial dependence, then the level shift acts as a spatially varying potential $U(\vec{r})$, and the atom feels its gradient $-\vec{\nabla} U(\vec{r})$ as a force.

This mirror-induced force is a peculiar manifestation of the mechanical effects of light. Forces due to applied light fields were first demonstrated experimentally by Lebedev [11], and the recoil of an absorbed photon on an atom was observed by Frisch who deflected an atomic beam with incoherent light 12. With the advent of the laser, such forces have found many important applications, from decelerating, cooling and trapping atoms to optical tweezers in biology [13.

Mirror-induced forces on individual atoms were first considered in connection with cavity-QED experiments, where their use has been proposed for trapping atoms in an optical resonator 14, 15. It is this kind of binding force which we observe in the experiment reported here. A single, trapped and laser-excited ion is an ideal system for this observation, as its position can be controlled on the nanometer scale [16, 17, 18], and interac- tion with a distant mirror has already been demonstrated [10, 16, 19]. These earlier experiments detected the effect of a mirror on the internal, electronic state of an ion. In contrast, our new study reveals directly the action on the ion's motional degree of freedom, whereby we have a new level of control over the total state of the atom and new possibilities for its manipulation. While the previous observations [10] have implications, e.g., for precision spectroscopy, our new results are more relevant for studies of single ions in optical cavities, for their cooling [20] and their application in quantum information processing.

A mirror-induced energy shift of an excited state, like a modified spontaneous decay rate [16, 19], has an analogy in classical electrodynamics. The classical effect is used, for example, to modify the emission diagram of an antenna by reflectors, and it is well-known that such geometric modifications also change the resonance frequency 21. These effects can be treated in terms of radiation reaction only, i.e. in terms of the interaction with the reflected field. The quantum electrodynamic picture is quite different though, due to the presence of the vacuum field which also couples to the atom 22]. The concept of the vacuum field forms the prevailing language in the field of experimental cavity QED, see for example Refs. 7, 8]. It was also used in the proposals to trap an atom in a resonator 14, 15] to which our experiment is closely related. Rigorously speaking, however, the vacuum field alone cannot account for spontaneous decay or its modification by reflectors, but radiation reaction must also contribute 23, 24, 25, 26]. The same is true for excitedstate level shifts. In fact, the degree to which vacuum fields and radiation reaction are seen to contribute depends upon the ordering of operators in the Heisenberg equations of motion, the choice of which has been called a "matter of taste" 22. In what follows we will use the concept of vacuum fields as a convenient language but without insisting on any particular distinction between vacuum fields and radiation reaction.

In our experiment with a single trapped $\mathrm{Ba}^{+}$ion, a fraction $\epsilon$ of its fluorescence light is retro-reflected and focussed back upon the emitting particle (see Fig. 1 below). For this situation, the model by Dorner [9] predicts 
an energy shift of the excited level by

$$
U(z)=-\hbar \frac{\epsilon \Gamma}{2} \sin (2 k z)
$$

Here $\Gamma$ is the decay rate of the excited level, $k=2 \pi / \lambda$ is the wave vector of the light with wavelength $\lambda$, and $z$ is the position of the mirror with respect to the ion. Depending on $z$, this potential creates different mechanical effects: around $\sin (2 k z)=0$, a force is exerted on the ion which points either towards or away from the mirror; around $\sin (2 k z)= \pm 1$, a binding $(+1)$ or antibinding $(-1)$ potential is formed. Since the atom feels the mirror-induced potential only whilst it resides in the excited level, the forces are scaled by the probability $P_{e}$ for the atom to be in that state. The maximum force at $\sin (2 k z)=0$ is therefore calculated as $P_{e} \hbar k \epsilon \Gamma$. The binding / anti-binding potential at $\sin (2 k z)= \pm 1$ is characterised by the oscillation frequency which an otherwise force-free atom would have in the respective potential well, $\omega_{v a c}=\left(2 P_{e} \epsilon \Gamma \hbar k^{2} / m\right)^{1 / 2}$, with atomic mass $m$. For an ion which is already confined with trap frequency $\omega_{\text {trap }}$ (typically around $2 \pi \times 1 \mathrm{MHz}$ ), the potential $U(z)$ of Eq. (II) adds to the trapping potential, thus changing the trap frequency according to $\omega_{\text {trap }}^{\prime}=\left(\omega_{\text {trap }}^{2}+\omega_{\text {vac }}^{2} \sin (2 k z)\right)^{1 / 2}$. Since the deviation $\delta \omega_{\text {trap }}=\omega_{\text {trap }}^{\prime}-\omega_{\text {trap }}$ is small, it is well approximated by

$$
\delta \omega_{\text {trap }}(z)=\frac{P_{e} \epsilon \Gamma \hbar k^{2}}{m \omega_{\text {trap }}} \sin (2 k z)
$$

It is this change of the trap frequency, a direct mechanical action, which we measure in the experiment. We emphasize that the level shift $U(z)$ producing this extra trapping force is caused by the presence of a single distant mirror and the associated modification of vacuum and radiation reaction fields. This distinguishes our observations from the recently demonstrated trapping of atoms in excited high-finesse optical resonators, where the mechanical action arises not only from reradiated photons, but also from the externally excited resonator mode [27, 28].

The experimental setup is shown in Fig. 1] The counting signal on the PMT exhibits high-contrast interference fringes as the ion-mirror distance is varied 16. This interference signal follows the $-\cos (2 k z)$ dependence of the modified $493 \mathrm{~nm}$ decay rate [9], such that the midpoints of the slopes correspond to $\sin (2 k z)= \pm 1$, i.e. to the maximum binding or anti-binding potential, as described above (see also Fig. [5 below). The trap frequency is measured by spectrally analysing the PMT signal. It contains a spectral component at the trap frequency, around $1 \mathrm{MHz}$, because the oscillation of the ion creates an intensity modulation of the scattered light. The signal on the spectrum analyser has, to good approximation, a Lorentzian line shape with width $\Delta f$ of about $500 \mathrm{~Hz}$. After a few seconds of averaging, the centre frequency of the line is determined with less than $10 \mathrm{~Hz}$ inaccuracy.

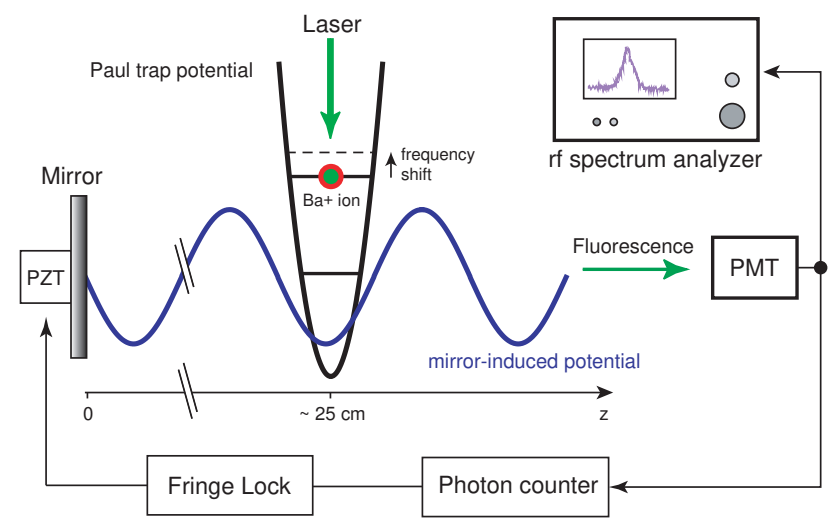

FIG. 1: Principle of the experiment. A single trapped ${ }^{138} \mathrm{Ba}^{+}$ ion is laser-excited at $493 \mathrm{~nm}$. A retro-reflecting mirror $25 \mathrm{~cm}$ away from the trap and a lens (not shown) are arranged such that they image the ion onto itself. The $493 \mathrm{~nm}$ fluorescence is detected by a photomultiplier (PMT). Its intensity modulation, i.e. the motional sideband due to the ion's oscillation in the trap, is recorded with a spectrum analyser. From the three different trap vibrations, we observe the one at the lowest frequency, $\omega_{x} \approx 2 \pi \times 1.02 \mathrm{MHz}$, whose orientation is at about $54^{\circ}$ to the optical axis. The deviation of the mean count rate from a chosen offset value is used in a feedback loop to control the position of the mirror such that the ion stays at a given point on an interference fringe to within $\sim 10 \mathrm{~nm}$. In the diagram, PZT stands for piezomechanical translator. The feedback loop has an integration time of about $1 \mathrm{~s}$ and compensates for slow drifts of the ion-mirror distance but not for the ion's oscillation in the trap. By switching the sign of the feedback gain, we choose between the positive and negative slopes of the interference fringes. More details of the setup are found in Refs. [10, 16].

Fig. 2 shows two spectra which were recorded directly one after the other, with the ion positioned on the midpoints of a positive and negative slope of the interference signal, respectively. The shift is clearly visible and amounts to $310 \mathrm{~Hz}$ in this case. The value is within the range expected from Eq. 2] which predicts around $350 \mathrm{~Hz}$, taking typical values for our experiment $P_{e} \approx 7 \%, \epsilon \approx 1.5 \%, \lambda=493 \mathrm{~nm}$, and $\Gamma=2 \pi \times 15.4 \mathrm{MHz}$. The corresponding value of $\omega_{v a c}$ is about $20 \mathrm{kHz}$, larger than the photon recoil frequency of $6 \mathrm{kHz}$. We emphasize that no changes are made to the setup between the recording of the two spectra, apart from translating the distant mirror by $\lambda / 4$.

To measure the value of the frequency shift for a particular set of parameters, we record about 60 spectra, alternating between the two slopes. Each spectrum is fitted by a Lorentzian, and the centre frequency is plotted. An example is shown in Fig. 3. While the trap frequency itself varies due to slow drifts of the trap drive intensity and due to thermal effects, a constant difference is observed between the values measured on the two slopes. The precise value of the shift depends on details of the experiment such as the settings of the lasers, their directions, and the fine alignment of the back-reflecting 


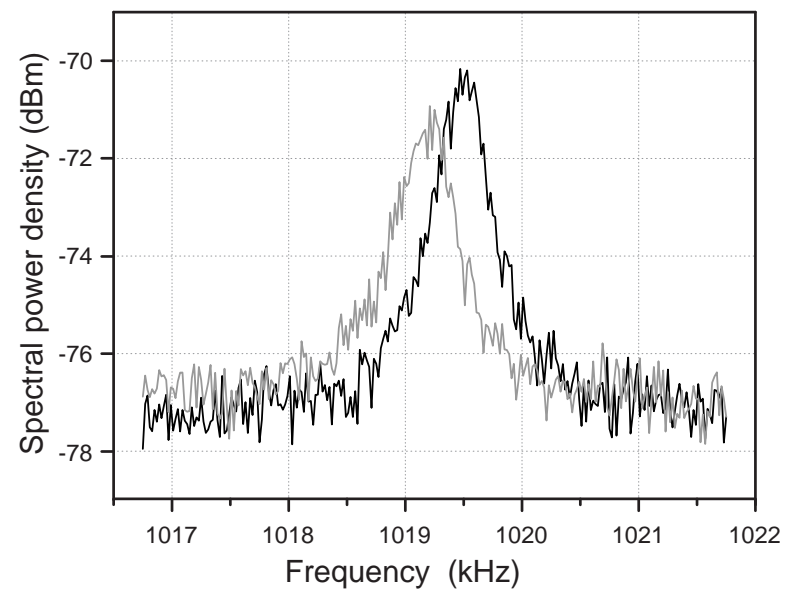

FIG. 2: Signal on the spectrum analyser for the ion positioned on the positive (right curve) and negative slope (left curve) of the interference signal. The centre frequency of a Lorentzian fit to the data is taken as the trap frequency. The broadening of the lines is a consequence of the ongoing laser cooling of the ion, and the width represents the steady-state cooling and heating rate [30]. The measured values agrees well with the expectation [31]. The size of the Lorentzian curve above the Poissonian noise level is observed to vary between 2 and $10 \mathrm{~dB}$ and serves as a measure of the amplitude of the ion's oscillation in the trap.

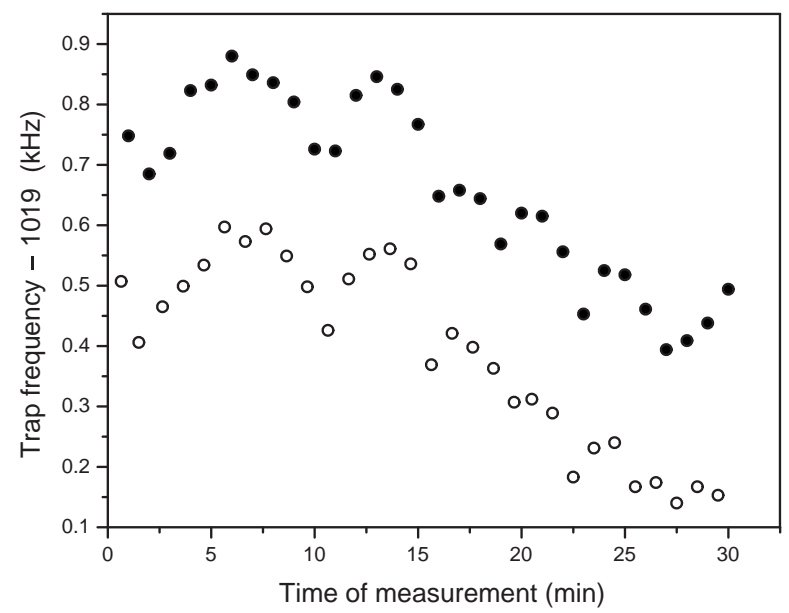

FIG. 3: Trap frequency measured on the positive (full circles) and negative slope (open circles) of the interference signal, vs. measurement time.

mirror. We observe values between 50 and $350 \mathrm{~Hz}$, all within the range expected from Eq. 22 It is important to note that we always find the higher trap frequency on the positive slope of the interference fringes (count rate vs. ion-mirror distance), in agreement with the theoretical prediction [9].

As shown in Eq. 22 the trap frequency shift depends on the laser parameters through the probability $P_{e}$ with which the ion is found in the excited state. This dependence has been measured by recording the maximum shift for different laser parameters. The mean fluores- cence level, at the midpoint of the interference fringes, serves as an indicator of $P_{e}$, to which it is strictly proportional. The result is displayed in Fig. 目 The data agree well with the expected linear dependence. A further test is the dependence of the trap frequency on the position of the mirror. When we shift the ion between the maxima and minima of the interference fringes, we find the result shown in Fig. 5 The sinusoidal variation predicted by Eq. (2) is clearly observed.

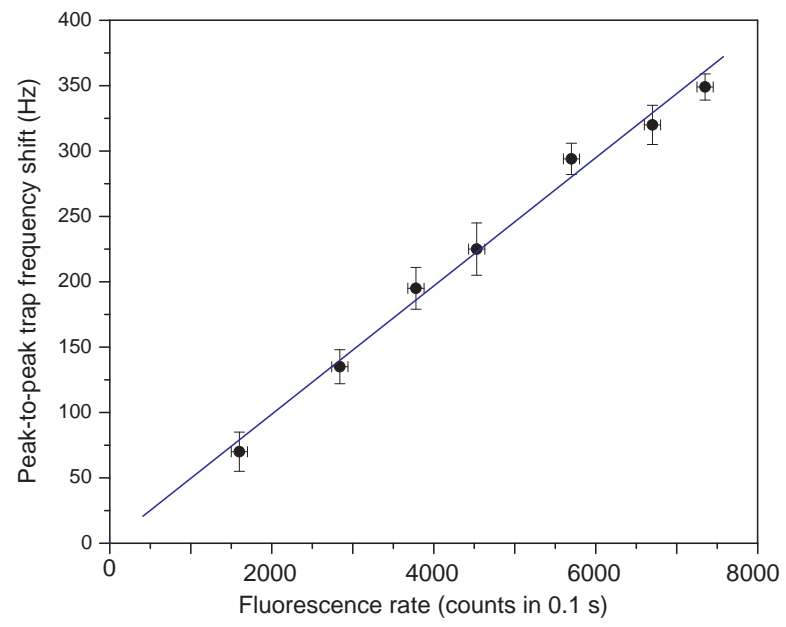

FIG. 4: Measured dependence of trap frequency variation on excited state population $P_{e}$. The peak-to-peak difference, between the midpoints of the two slopes of the interference fringes, is plotted vs. the mean count rate. 10000 counts correspond to $P_{e} \approx 0.1$. The line is a linear fit.

One may construct a semi-classical explanation for the observed mechanical action, analogous to the intuitive picture in [9] that the level shift corresponds to the energy of the atomic dipole in the light field returning from the mirror. When a maximum in the interference fringes is observed at the PMT, the returning light stimulates additional radiation towards the PMT, thus creating a small recoil towards the mirror. Conversely, a minimum in the interference fringes corresponds to radiation returning from the mirror being predominantly absorbed, which therefore leads to a force away from the mirror. However, the quantum mechanical properties of the electromagnetic field are needed to explain quantitatively the spontaneous emission rate from an atom [22, 26, 29]. Therefore the concepts of vacuum fields and radiation reaction, as presented in the introduction, are felt to be the most accurate way of describing our experimental findings.

We emphasize that it is a distant and passive optical element which introduces a controlled, position-dependent mechanical action on the atom in our experiment. In general, any dielectric optical element which back-reflects the light scattered from nearby atoms shifts the excited levels, modifies the transition frequencies, and acts on the motional state. Therefore our study is also relevant for technological applications of single trapped atoms 


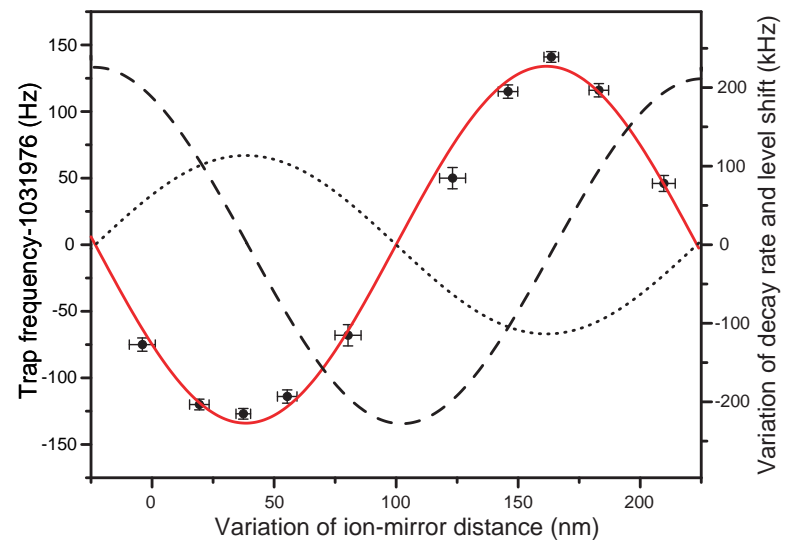

FIG. 5: Measured variation of the trap frequency with ionmirror distance (data points). The distance is adjusted by varying the offset level in the feedback loop to the PZT. This data set showed particularly small drifts of the trap frequency. The solid line is a fit to the data. The dashed line shows the corresponding, calculated variation of the spontaneous decay rate on the S-P transition, and the dotted line is the shift of the excited level, i.e. the vacuum potential divided by $\hbar$. The dashed and dotted lines use the right-hand vertical scale. The calculated maximum force, acting when the atom is positioned on a maximum or minimum of the dashed curve, corresponds to an acceleration of $\sim 100 \mathrm{~g}$. and ions, in particular when they are combined with high-finesse optical cavities. On the other hand, since energy shifts of individual levels accumulate in time to phase shifts of the atomic wavefunction, their control, as demonstrated here, may become useful for the manipulation of optical phases in applications of single atoms or ions for quantum state engineering or quantum information processing. Another possible application would be "vacuum optical tweezers" which exert forces on a laserexcited molecule just by an arrangement of microscopic mirrors.

Acknowledgements. This work is supported by the Austrian Science Fund (FWF, SFB15), the European Commission (QUEST network, HPRNCT-2000-00121, QUBITS network, IST-1999-13021), and the "Institut für Quanteninformation GmbH". P. B. thanks A. Allahverdyan and K. Lamonova for stimulating discussions. The authors thank Uwe Dorner for clarifying remarks.
* Present address: Institut de Ciències Fotòniques (ICFO), Barcelona, Spain.

[1] H. B. G. Casimir, D. Polder, Phys. Rev. 73, 360 (1948).

[2] V. L. Lyouboshitz, Sov. Phys.-JETP 25, 612-617 (1967).

[3] H. Morawitz, Phys. Rev. 187, 1792-1796 (1969).

[4] G. Barton, Proc. R. Soc. Lond. A 410, 141-174 (1987).

[5] D. Meschede, W. Jhe, E. A. Hinds, Phys. Rev. A 41, 1587-1596 (1990).

[6] E. A. Hinds, Adv. At. Mol. Opt. Phys. 28, 237-289 (1991).

[7] D. J. Heinzen and M. S. Feld, Phys. Rev. Lett. 59, 26232626 (1987).

[8] M. Brune, P. Nussenzveig, F. Schmidt-Kaler, F. Bernardot, A. Maali, J. M. Raimond, S. Haroche, Phys. Rev. Lett. 72, 3339-3342 (1994).

[9] U. Dorner and P. Zoller, Phys. Rev. A 66, 023816 (2002).

[10] M. A. Wilson, P. Bushev, J. Eschner, F. Schmidt-Kaler, C. Becher, and R. Blatt, U. Dorner, Phys. Rev. Lett. 91, 213602 (2003).

[11] P. N. Lebedev, Ann. d. Physik 6, 433-458 (1901).

[12] R. Frisch, Z. Phys. 86, 42-48 (1933).

[13] K. Dholakia, G. Spalding and M. MacDonald, Physics World 15, 31-35 (2002).

[14] S. Haroche, M. Brune, and J. M. Raimond, Europhys. Lett. 14, 19-24 (1991).

[15] C. Schön and J. I. Cirac, Phys. Rev. A 67, 043813 (2003).

[16] J. Eschner, Ch. Raab, F. Schmidt-Kaler, and R. Blatt, Nature 413, 495-498 (2001).

[17] G. R. Guthöhrlein, M. Keller, K. Hayasaka, W. Lange, and H. Walther, Nature 414, 49-51 (2001).
[18] A. B. Mundt, A. Kreuter, C. Becher, D. Leibfried, J. Eschner, F. Schmidt-Kaler, R. Blatt, Phys. Rev. Lett. 89, 103001 (2002).

[19] A. Mundt, PhD thesis, Innsbruck 2003.

[20] P. Domokos, H. Ritsch, J. Opt. Soc. Am. B, 20, 10981130 (2003).

[21] see for example Blake, L. V., "Antennas" (John Wiley and Sons, 1966).

[22] Milonni, P. W., "The Quantum Vacuum" (Academic, San Diego, 1994).

[23] P. W. Milonni, J. R. Ackerhalt, W. A. Smith, Phys. Rev. Lett. 31, 958-960 (1973).

[24] J. R. Ackerhalt, P. L. Knight, J. H. Eberly, Phys. Rev. Lett. 30, 456 (1973).

[25] J. Dalibard, J. Dupont-Roc, C. Cohen-Tannoudji, J. Phys. (Paris) 43, 1617 (1982).

[26] E. A. Hinds and V. Sandoghdar, Phys. Rev. A 43, 398403 (1991).

[27] P. W. H. Pinkse, T. Fischer, P. Maunz, and G. Rempe, Nature 404, 365-368 (2000).

[28] C. J. Hood, T. W. Lynn, A. C. Doherty, A. S. Parkins, and H. J. Kimble, Science 287, 1447-1453 (2000).

[29] W. Heitler, "The Quantum Theory of Radiation", 3rd. ed., Oxford University Press, London, 1954.

[30] J. I. Cirac, R. Blatt, A. S. Parkins, and P. Zoller, Phys. Rev. A 48, 2169-2181 (1993).

[31] Ch. Raab, J. Eschner, J. Bolle, H. Oberst, F. SchmidtKaler, R. Blatt, Phys. Rev. Lett. 85, 538-541 (2000). 